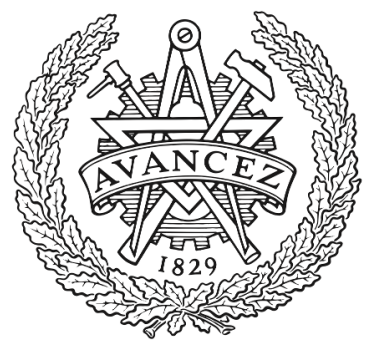

CHALMERS

UNIVERSITY OF TECHNOLOGY

\title{
On the Impact of Intercore Skew on Joint-Core Carrier-Phase Estimation
}

Downloaded from: https://research.chalmers.se, 2023-04-26 13:17 UTC

Citation for the original published paper (version of record):

Alfredsson, A., Agrell, E., Wymeersch, H. et al (2019). On the Impact of Intercore Skew on Joint-Core Carrier-Phase Estimation. European Conference on Optical Communication, ECOC(CP765). http://dx.doi.org/10.1049/cp.2019.0859

N.B. When citing this work, cite the original published paper. 


\title{
ON THE IMPACT OF INTERCORE SKEW ON JOINT-CORE CARRIER-PHASE ESTIMATION Arni F. Alfredsson ${ }^{1 *}$, Erik Agrell, Henk Wymeersch ${ }^{1}$, and Magnus Karlsson ${ }^{2}$

\author{
${ }^{I}$ Department of Electrical Engineering, Chalmers University of Technology, Gothenburg, Sweden \\ ${ }^{2}$ Department of Microtechnology and Nanoscience, Chalmers University of Technology, Gothenburg, Sweden \\ *E-mail: arnia@chalmers.se
}

Keywords: carrier-phase estimation, coherent communication, multicore fiber, skew, space-division multiplexing

\begin{abstract}
The impact of intercore skew on joint-core carrier-phase estimation in multicore-fiber transmission is studied. It is shown that the performance degradation is not dependent on the combined linewidth of the light-source laser and the local oscillator, but rather the ratio between the two linewidths.
\end{abstract}

\section{Introduction}

Phase noise is an inherent issue in coherent fiber-optic communication systems. This impairment is typically compensated using carrier-phase estimation (CPE) algorithms through digital signal processing (DSP). In general, CPE can be implemented using pilot symbols [1, 2] or in a blind fashion, i.e., without the use of pilots [3, 4]. Furthermore, CPE has been studied recently in the context of space-division multiplexed (SDM) transmission [2, 5-8]. Generally speaking, SDM attempts to facilitate the upscaling of optical networks in a costefficient manner through the sharing of hardware and other resources, as well as the use of specialized fibers such as multicore fibers (MCFs) [9]. In particular, the sharing of lasers among the different cores in MCFs gives rise to correlated phase noise across the spatial channels.

The sharing of lasers has led to methods such as selfhomodyne detection [10] and master-slave CPE [8] that exploit the spatial correlation to reduce the required computational complexity for CPE. Moreover, CPE that entails joint-core processing has been demonstrated [5], showing improved system tolerance to phase noise compared to per-core CPE thanks to the spatial correlation in the phase noise. A similar method has been demonstrated for frequency-comb-based wavelengthdivision multiplexed systems [2].

A phenomenon pertinent to CPE is intercore skew [11], which occurs in MCF transmission due to differences in refractive indices between the cores and is typically on the order of $100 \mathrm{ps} / \mathrm{km}$ up to $\mathrm{ns} / \mathrm{km}$ [12]. Unless compensated optically, it causes the phase noise to decorrelate across the cores, which reduces the potency of schemes that exploit the correlation. This problem has been investigated for self-homodyne detection [13] and master-slave CPE [14], where it was shown that skew can be detrimental to the performance of these techniques. However, the impact of intercore skew has not been studied for joint-core CPE. The questions addressed in this paper can thus be formulated as follows: (i) Can an established scheme for joint-core CPE that was not originally designed to account for skew be modified to become effective in the presence of intercore skew, and (ii) if so, when is it beneficial and how much can performance be improved over CPE on a per-core basis?

\section{System Model}

Consider pilot-aided dual-polarization (DP) transmission at a symbol rate of $R_{\mathrm{s}}$ through $D$ cores of an uncoupled-core, single-mode MCF. The light-source laser and local oscillator (LO) are assumed to be shared between all cores, which gives rise to a common phase noise in all spatial channels. Standard DSP steps on a per-core basis are assumed to have taken place, such as dispersion compensation, adaptive equalization, frequency-offset compensation, frame synchronization, and resampling. The equalization is assumed to be implemented with a phase-immune equalizer [15], and perfect knowledge of the intercore skew has been obtained through the frame synchronization. Interpolarization skew is neglected, but due to the presence of intercore skew, the received signals are assumed to be realigned in the receiver DSP. Finally, the values and positions of pilot symbols are assumed known to the receiver, and data symbols are modelled as random variables that take values in a set of complex constellation points. With these assumptions in place, the discrete-time complex baseband model is written as

$$
r_{w, k}^{(i)}=s_{w, k}^{(i)} e^{j \theta_{k}^{(i)}}+n_{w, k}^{(i)},
$$

where $w \in\{\mathbf{x}, \mathbf{y}\}$ is the polarization index, $k=1, \ldots, N$ is the time index, and $i=1, \ldots, D$ is the core index. Moreover, $r_{w, k}^{(i)}$ is the received sample, $s_{w, k}^{(i)}$ is the transmitted symbol, $\theta_{k}^{(i)}$ is the phase-noise sample, and $n_{w, k}^{(i)}$ is the additive white Gaussian noise sample. In [5], $\theta_{k}^{(i)}$ was modelled as the sum of a single random-walk component, common to all spatial channels, and a channel-specific random walk. This model was found effective for MCF transmission in the absence of skew, which was realized through careful path alignment in the experimental setup.

In this paper, the channel-specific random-walk component is considered constant throughout the transmitted block. To 


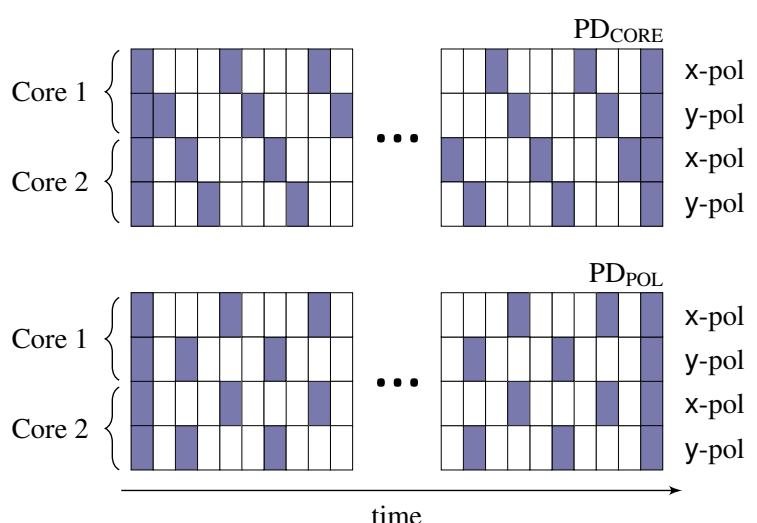

Fig. 1 The considered pilot-symbol distributions, $\mathrm{PD}_{\mathrm{CORE}}$ (top) and $\mathrm{PD}_{\mathrm{POL}}$ (bottom), in the case of two cores and approximately $25 \%$ pilot rate for illustration purposes. The dark and white blocks correspond to pilot and data symbols, respectively.

account for intercore skew, the phase noise in each channel is assumed to be the sum of two components corresponding to the phase noise of the light-source laser and LO. Since the received blocks are realigned in the receiver DSP, the light-source laser phase noise will be aligned in all channels, but the LO phase noise becomes misaligned. Hence,

$$
\theta_{k}^{(i)}=\theta_{\mathrm{LS}, k}+\theta_{\mathrm{LO}, k+\xi_{i}},
$$

where $\xi_{i} \geq 0$ is the intercore skew with respect to the core whose corresponding signal propagates with the smallest velocity. Moreover, $\theta_{\mathrm{LS}, k}$ and $\theta_{\mathrm{LO}, k}$ are modelled as random walks, i.e., $\theta_{\mathrm{LS}, k}=\theta_{\mathrm{LS}, k-1}+\dot{\theta}_{\mathrm{LS}, k}$ and $\theta_{\mathrm{LO}, k}=\theta_{\mathrm{LO}, k-1}+$ $\dot{\theta}_{\mathrm{LO}, k}$, with $\theta_{\mathrm{LS}, 1}$ and $\theta_{\mathrm{LO}, 1}$ drawn uniformly on $[0,2 \pi)$, whereas $\dot{\theta}_{\mathrm{LS}, k}$ and $\dot{\theta}_{\mathrm{LO}, k}$ are zero-mean Gaussian random variables with variances $2 \pi \Delta \nu_{\mathrm{LS}} / R_{\mathrm{s}}$ and $2 \pi \Delta \nu_{\mathrm{LO}} / R_{\mathrm{s}}$, respectively. Furthermore, $\Delta \nu_{\mathrm{LS}}$ and $\Delta \nu_{\mathrm{LO}}$ are the linewidths of the light-source laser and LO.

\section{CPE Strategy}

The model-based algorithm for joint-channel CPE used in [5] is considered. It is designed based on a multidimensional random-walk model for the phase noise across all channels to perform iterative CPE and symbol detection, using pilot symbols to bootstrap the iterations. The CPE is implemented through an extended Kalman smoother, and hence, a covariance matrix $\mathbf{Q}$ is utilized that describes the correlation in the phase noise across the channels.

For the phase-noise model in (2) it can be shown that the skew-induced intercore phase differences are not random walks, but rather moving-average processes [16, Ch. 10.4]. As a consequence, the phase noise across all channels cannot be described as a multidimensional random walk with a covariance matrix $\mathbf{Q}$. To handle this issue, the CPE algorithm is modified to include a numerical optimization of $\mathbf{Q}$ that minimizes the mean squared error (MSE) of the resulting phase-noise estimates.
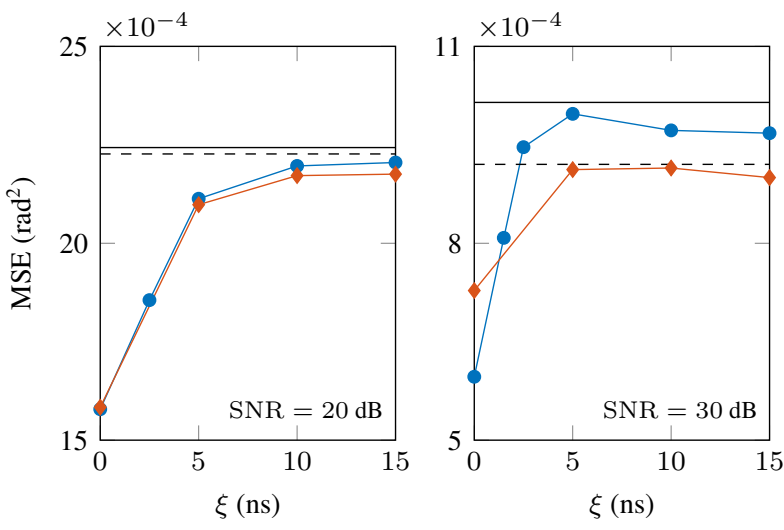

$$
\begin{aligned}
& -\mathrm{JCPE}_{\mathrm{CORE}} \& \mathrm{PD}_{\mathrm{CORE}} \multimap \mathrm{JCPE}_{\mathrm{CORE}} \& \mathrm{PD}_{\mathrm{POL}} \\
& -\mathrm{JCPE}_{\mathrm{POL}} \& \mathrm{PD}_{\mathrm{CORE}}---\mathrm{JCPE}_{\mathrm{POL}} \& \mathrm{PD}_{\mathrm{POL}}
\end{aligned}
$$

Fig. 2 MSE versus skew for JCPE $\mathrm{CORE}_{\mathrm{C}}$ and JCPE $\mathrm{POL}_{\mathrm{P}}$ with both pilot distributions, illustrating that for a specific pilot distribution, joint-core CPE performs similarly or better than per-core CPE regardless of skew.

\section{Numerical Results}

Uncoded DP transmission of 256-ary quadrature amplitude modulation (256QAM) is considered at 20 GBaud through two cores of an ideally uncoupled MCF. Numerical results are obtained using Monte Carlo simulations, the system model in (1)-(2), and the modified CPE algorithm described in the previous section, running 1 iteration. The results comprise phase-noise-estimate MSEs, bit error rates (BERs), and achievable information rates (AIRs) for different signal-tonoise ratios (SNRs), amounts of skew denoted by $\xi \triangleq \mid \xi_{1}-$ $\xi_{2} \mid$, and light-source linewidth to LO linewidth ratios denoted by $R_{\mathrm{LW}} \triangleq \Delta \nu_{\mathrm{LS}} / \Delta \nu_{\mathrm{LO}}$. The AIR is obtained by computing the generalized mutual information for mismatched decoding and subtracting the pilot rate, which is fixed to $1 \%$ for all the results. Unless specified otherwise, the combined laser linewidth denoted by $\Delta \nu_{\mathrm{C}} \triangleq \Delta \nu_{\mathrm{LS}}+\Delta \nu_{\mathrm{LO}}$ is fixed at 200 $\mathrm{kHz}$. Finally, frames of 100000 symbols across all channels are transmitted at least 10 times to obtain the results.

Two CPE strategies are compared: (i) joint-core CPE, which entails joint processing across all cores and polarizations, denoted by $\mathrm{JCPE}_{\mathrm{CORE}}$, and (ii) joint-polarization CPE on a per-core basis, denoted by $\mathrm{JCPE}_{\mathrm{POL}}$, whose performance is independent of $\xi$. Thus, by comparing the two strategies, it can be determined how much $\xi$ is tolerable for $\mathrm{JCPE}_{\mathrm{CORE}}$ to provide benefits over JCPE $\mathrm{POL}_{\mathrm{PO}}$. Both strategies are implemented using the CPE algorithm described in the previous section. As the algorithm is highly dependent on the placement of pilot symbols, two pilot distributions are considered, denoted by $\mathrm{PD}_{\mathrm{CORE}}$ and $\mathrm{PD}_{\mathrm{POL}}$ and illustrated for approximately $25 \%$ pilot rate in Fig. 1. The former distribution is found to be beneficial for JCPE $\mathrm{CORE}_{\mathrm{C}}$ in the presence of low or negligible $\xi$, particularly at high SNRs, whereas the latter is more beneficial for 


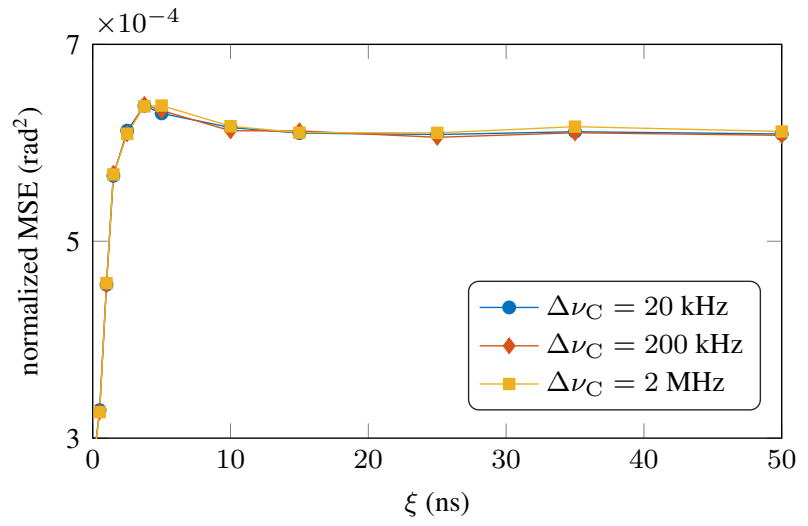

Fig. 3 Normalized MSE versus skew for different combined light-source-laser and LO linewidth, showing that the performance of the CPE algorithm in the presence of skew is independent of the combined laser linewidth.

$\mathrm{JCPE}_{\mathrm{POL}}$, regardless of $\xi$ and SNR. Unless mentioned otherwise, JCPE $\mathrm{JORE}_{\mathrm{CO}}$ is used with $\mathrm{PD}_{\mathrm{CORE}}$ and $\mathrm{JCPE}_{\mathrm{POL}}$ is used with $\mathrm{PD}_{\mathrm{POL}}$.

Fig. 2 presents the MSE performance of $\mathrm{JCPE}_{\mathrm{CORE}}$ and $\mathrm{JCPE}_{\mathrm{POL}}$ with both of the considered pilot distributions for $R_{\mathrm{LW}}=1$, which implies that $\Delta \nu_{\mathrm{LS}}=\Delta \nu_{\mathrm{LO}}=100 \mathrm{kHz}$. As evident from the plots, JCPE $\mathrm{C}_{\mathrm{CORE}}$ performs similarly to or better than $\mathrm{JCPE}_{\mathrm{POL}}$ for all $\xi$ when both strategies use the same pilot distribution. It is found that the performance does not change much with greater values of $\xi$ than what is shown.

Fig. 3 shows the MSE performance of JCPE $\mathrm{CORE}_{\text {versus } \xi \text { for }}$ different values of $\Delta \nu_{\mathrm{C}}$ and $R_{\mathrm{LW}}=1$, where the MSE has been normalized for each curve in order to facilitate comparison. All the curves are essentially identical, which indicates that $\Delta \nu_{\mathrm{C}}$ does not influence the tolerance of $\mathrm{JCPE}_{\mathrm{CORE}}$ to $\xi$. An intuitive explanation for this is that the amount of skew-induced intercore phase differences for a given $\xi$, relative to the amount of absolute phase noise in each channel, does not change if $\Delta \nu_{\mathrm{C}}$ is changed. In other words, the spatial correlation in the phase noise across the cores does not depend on $\Delta \nu_{\mathrm{C}}$. In contrast, it is found that $R_{\mathrm{LW}}$ does indeed affect the spatial correlation, which in turn influences the effectiveness of JCPE $\mathrm{CORE}_{\mathrm{C}}$.

To demonstrate the influence of $R_{\mathrm{LW}}$ on the efficacy of JCPE $_{\text {CORE }}$, Fig. 4 shows BER and AIR versus SNR, comparing the performance of $\mathrm{JCPE}_{\mathrm{CORE}}$ and $\mathrm{JCPE}_{\mathrm{POL}}$ for $\xi=50 \mathrm{~ns}$ and different values of $R_{\mathrm{LW}}$. As a reference, the performance of JCPE $\mathrm{CORE}_{\mathrm{C}}$ in the absence of skew $(\xi=0)$, as well as in the ideal case of no phase noise, is included. As can be expected from Fig. 2, JCPE $\mathrm{CORE}_{\mathrm{C}}$ and $\mathrm{JCPE}_{\mathrm{POL}}$ perform similarly when $R_{\mathrm{LW}}=1$. However, when $R_{\mathrm{LW}}=10, \mathrm{JCPE}_{\mathrm{CORE}}$ sees better performance since the spatial correlation in the phase noise across the cores is greater than in the case of $R_{\mathrm{LW}}=1$. It is also worth noting that the performance of $\mathrm{JCPE}_{\mathrm{CORE}}$ for $\xi=0$ is identical to the case where $R_{\mathrm{LW}} \rightarrow \infty$ and $\xi>0$, i.e., when the phase noise comes solely from the light-source laser. In this case, the phase noise is fully correlated across the cores and $\mathrm{JCPE}_{\mathrm{CORE}}$ sees the biggest benefits, providing approximately
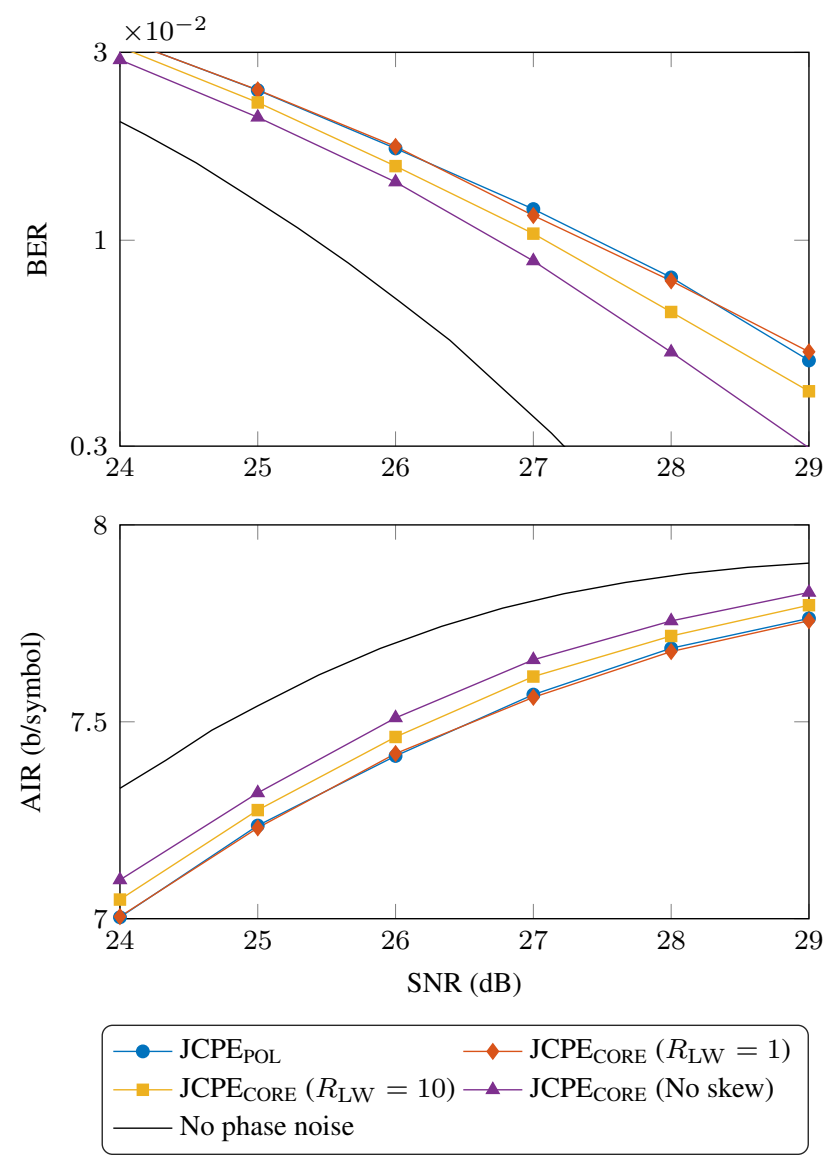

Fig. 4 BER and AIR in b/symbol per polarization versus SNR for 256QAM, illustrating how the performance of joint-core CPE improves when the LO linewidth is less than the lightsource linewidth.

a $0.1 \mathrm{~b} /$ symbol gain in AIR per polarization and up to a $40 \%$ reduction in BER compared with $\mathrm{JCPE}_{\mathrm{POL}}$ for the tested SNRs.

\section{Conclusions}

This paper investigated the effects of intercore skew on the performance of an established joint-channel CPE algorithm, which was originally not designed to account for skew. It was shown that through a small modification to the algorithm, joint-core CPE performs similarly to or better than per-core CPE in the presence of skew. More specifically, when intercore skew is significant, the benefits of joint-core CPE depend mainly on the ratio between the light-source linewidth and the LO linewidth, but not on the combined laser linewidth. Assuming that the received signals are realigned in the receiver DSP after transmission, joint-core CPE is more beneficial if the LO has less linewidth than the light-source laser. Up to $0.1 \mathrm{~b} / \mathrm{symbol}$ gain in AIR per polarization and a BER reduction of $40 \%$ are obtained by joint-core CPE compared to per-core CPE for 256QAM transmission through two cores with $200 \mathrm{kHz}$ combined laser linewidth in the presence of significant skew. If more than two cores are jointly processed, further gains can be expected. 


\section{References}

[1] Colavolpe, G., Barbieri, A., Caire, G.: 'Algorithms for iterative decoding in the presence of strong phase noise', IEEE J. Sel. Areas Commun., 2005, 23, (9), pp. 17481757

[2] Millar, D.S., Maher, R., Lavery, D., et al.: 'Design of a $1 \mathrm{~Tb} / \mathrm{s}$ superchannel coherent receiver', J. Lightw. Technol., 2016, 34, (6), pp. 1453-1463

[3] Pfau, T., Hoffmann, S., Noé, R.: 'Hardware-efficient coherent digital receiver concept with feedforward carrier recovery for $M$-QAM constellations', J. Lightw. Technol., 2009, 27, (8), pp. 989-999

[4] Ip, E., Kahn, J.M.: 'Feedforward carrier recovery for coherent optical communications', J. Lightw. Technol., 2007, 25, (9), pp. 2675-2692

[5] Alfredsson, A.F., Agrell, E., Wymeersch, et al.: 'Pilotaided joint-channel carrier-phase estimation in spacedivision multiplexed multicore fiber transmission', J. Lightw. Technol., 2019, 37, (4), pp. 1133-1142

[6] Lundberg, L., Mazur, M., Lorences-Riesgo, A., et al.: 'Joint carrier recovery for DSP complexity reduction in frequency comb-based superchannel transceivers'. Proc. European Conf. Opt. Commun. (2017). p. Th.1.D.3

[7] Yankov, M.P., Barletta, L., Zibar, D.: 'Phase noise compensation for nonlinearity-tolerant digital subcarrier systems with high-order QAM', IEEE Photon. J., 2017, 9, (5)

[8] Feuer, M.D., Nelson, L.E., Zhou, X., et al.: 'Joint digital signal processing receivers for spatial superchannels', IEEE Photon. Technol. Lett., 2012, 24, (21), pp. 19571960

[9] Richardson, D.J., Fini, J.M., Nelson, L.E.: 'Spacedivision multiplexing in optical fibres', Nat. Photon., 2013, 7, (5), pp. 354-362

[10] Puttnam, B.J., Sakaguchi, J., Delgado Mendinueta, J.M., et al.: 'Investigating self-homodyne coherent detection in a 19 channel space-division-multiplexed transmission link', Opt. Express, 2013, 21, (2), pp. 1561-1566

[11] Luís, R.S., Puttnam, B.J., Delgado Mendinueta, J.M., et al.: 'Comparing inter-core skew fluctuations in multicore and single-core fibers'. Proc. Conf. Lasers and Electro-Opt. (2015). p. SM2L.5

[12] Puttnam, B.J., Luís, R.S., Rademacher, G., et al.: 'Characteristics of homogeneous multi-core fibers for SDM transmission', APL Photon. J., 2019, 4, (2), pp. 022804

[13] Luís, R.S., Puttnam, B.J., Delgado Mendinueta, J.M., et al.: 'Impact of spatial channel skew on the performance of spatial-division multiplexed self-homodyne transmission systems'. Proc. International Conf. Photon. Switching. (2015). pp. 37-39

[14] Lundberg, L.: 'Power Consumption and Joint Signal Processing in Fiber-Optical Comunication'. PhD thesis, Chalmers University of Technology, 2019

[15] Fatadin, I., Ives, D., Savory, S.J.: 'Blind equalization and carrier phase recovery in a 16-QAM optical coherent system', J. Lightw. Technol., 2009, 27, (15), pp. 3042-3049
[16] Miller, S.L., Childers, D.: 'Probability and Random Processes: With Applications to Signal Processing and Communications'. (Cambridge, MA, USA: Academic Press, 2nd edn., 2012) 